\title{
Arginine: an unusual dietary requirement of pre-B lymphocytes?
}

\author{
Tucker W. LeBien \\ Department of Laboratory Medicine and Pathology, University of Minnesota Cancer Center, Minneapolis, Minnesota, USA \\ J. Clin. Invest. 110:1411-1413 (2002). doi:10.1172/JCI200217210.
}

Mammalian B cell development originates primarily in the fetal liver, and continues in neonatal bone marrow (BM) and adult BM throughout life. An expansive body of published data has provided a detailed account of the patterns of gene expression that characterize discrete stages of murine and human B cell development $(1,2)$. Targeted disruption of genes encoding transcription factors, immunoglobulin heavy and/or light chains and a variety of kinases have revealed the contribution of these molecules to the traversal of distinct checkpoints in B cell development $(3,4)$. Cytokines (i.e., interleukins, chemokines, interferons, and colony-stimulating factors), the ECM, and stromal cell components of the BM microenvironment play a crucial role in regulating the survival, proliferation, differentiation, and death of B lineage cells (5). Immunologists are accustomed to thinking about the role of these well-studied molecules in B cell development, but what about the role of something so fundamental as an amino acid? An intriguing report by de Jonge and colleagues in this issue of the JCI (6) identifies the amino acid arginine as being unexpectedly important in a specific stage of murine B cell development.

\footnotetext{
Address correspondence to: Tucker W. LeBien, University of Minnesota Cancer Center, 420 Delaware Street SE, MMC 806, Minneapolis, Minnesota 55455, USA. Phone: (612) 626-1422; Fax: (612) 626-3941; E-mail: lebie001@umn.edu.

Conflict of interest: The author has declared that no conflict of interest exists.

Nonstandard abbreviations used: bone marrow (BM); B cell receptor (BCR); Bruton's tyrosine kinase (Btk); B cell linker protein (BLNK); stromal cell-derived factor-1 (SDF-1); $\mu$ heavy chains/surrogate light chains $(\mu / \mathrm{SLC})$.
}

\section{Arginine immune homeostasis}

Arginine is a precursor in the synthesis of proteins, nitric oxide, agmatine, creatinine, urea, and polyamines (7). The influence of dietary components on the integrity of the immune system has been studied for decades and a unique role for arginine in the maintenance of immune homeostasis has been proposed, particularly with respect to $T$ cell and macrophage function (8, and refs. cited therein). In a recent study, de Jonge et al. engineered transgenic mice expressing the enzyme arginase I under the control of the rat intestinal fatty-acid binding promoter and enhancer element (9). This transgene directed the overexpression of arginase I in the enterocytes of the small intestine. Analysis of several transgenic lines revealed that transient reduction of arginine plasma levels to $30-40 \%$ of control littermates had adverse affects on skin, muscle, and lymphoid development - albeit largely restricted to the initial three-week neonatal period. The affect on lymphoid development was most notably characterized by reduction in the size and number of Peyer's patches in gut-associated lymphoid tissue. The report by de Jonge and colleagues in this issue presents a more detailed analysis of lymphocyte development in arginase I transgenic mice, and describes the surprising observation that the transition of progenitor$B$ (pro-B) to precursor-B (pre-B) cells in $B M$ was particularly sensitive to a reduced availability of arginine (6). Specifically, in immunologic terms arginase I transgenic mice exhibited an inverse ratio of $\mathrm{B} 220^{+} / \mathrm{CD}_{4} 3^{+}$pro$\mathrm{B}$ cells to $\mathrm{B} 220^{+} / \mathrm{CD} 43^{-}$pre-B cells. This developmental block could reflect a failure of pro-B cells to dif- ferentiate to large pre-B cells and/or a failure of these pre-B cells to survive. Arginase I transgenic mice also had a quantitative decrease in B cell numbers in the spleen, lymph nodes, and Peyer's patches. However, the function of the mature B cells that did develop appeared intact. By contrast, once the arginase I transgenic mice were normalized for reduced body mass, there was no apparent affect on $\mathrm{T}$ cell development. How can these results be explained?

\section{Arginine requirement in $B$ cell development}

Although the disruption of $\mathrm{B}$ cell development at the pro- $\mathrm{B}$ to pre- $\mathrm{B}$ interface was reasonably convincing based on flow cytometric analysis of cell surface receptors, the expression of the pre-B cell receptor (BCR) and downstream signaling molecules was not reported. Previous studies have shown that $\mathrm{B} 220^{+} / \mathrm{CD} 19^{+} / \mathrm{CD} 43^{+} /$ $\mathrm{CD} 24^{+} / \mathrm{BP}^{+}$cells (fractions $\mathrm{C}$ and $\mathrm{C}^{\prime}$ in ref. 1) are characterized by the completion of variable to diversity/joining heavy chain rearrangements, and pairing of $\mu$ heavy chains with the surrogate light chain complex to form the pre-BCR (2). Furthermore, targeted disruption of genes encoding components of the pre-BCR or crucial signaling molecules downstream of the preBCR (e.g., Bruton's tyrosine kinase [Btk] or the $\mathrm{B}$ cell linker protein [BLNK]) disrupts B cell development at a stage very similar to the developmental block in the arginase I transgenic mice described by de Jonge and colleagues $(2,3)$. Analysis of the expression of the pre-BCR and downstream signaling molecules in arginase I transgenics is an obvious experiment that may prove productive. 

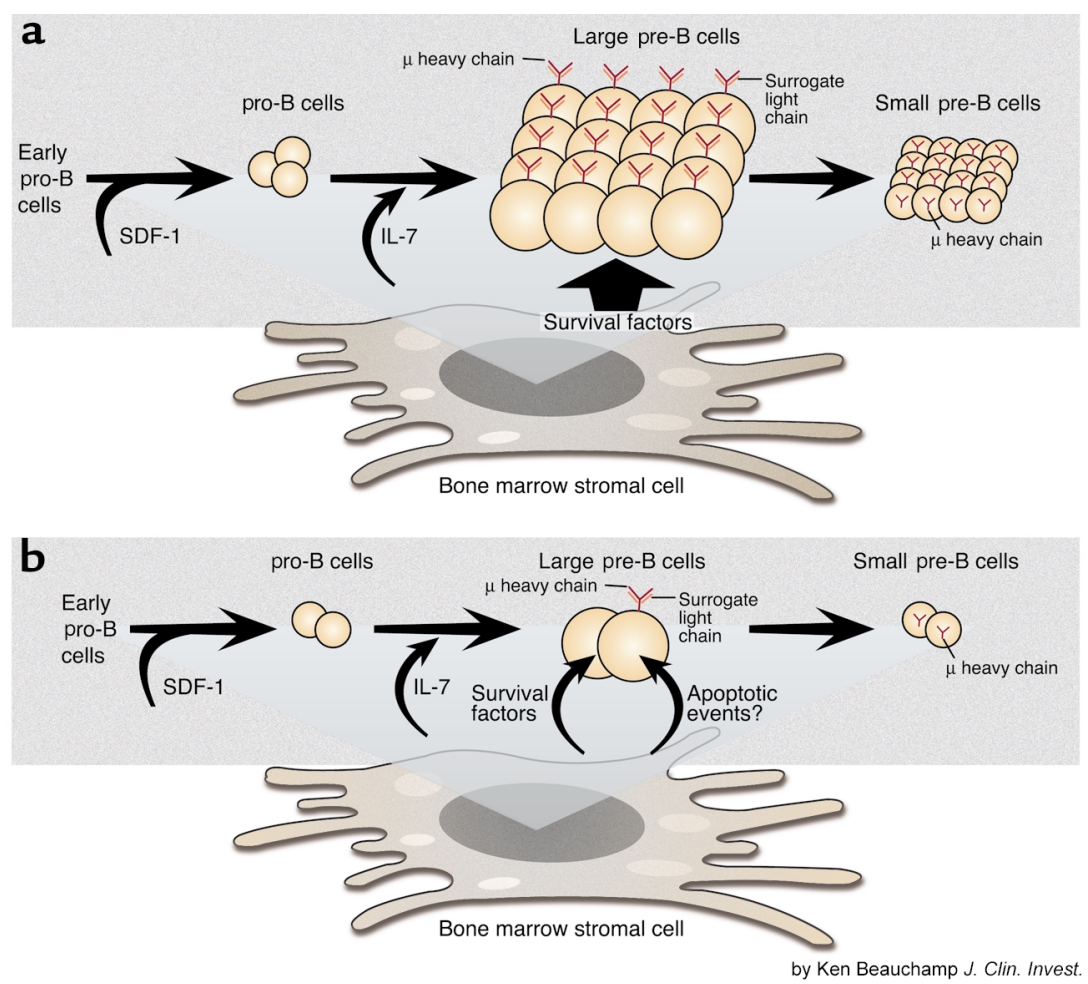

Figure 1

(a) B cell development in wild-type mice under normal steady state conditions. The pro-B, large pre- $B$, and small pre- $B$ cells correspond to fractions $C, C^{\prime}$, and $D$ (as discussed in ref. 1). Notably, large pre-B cells are enriched for cycling cells expressing $\mu$ heavy chains/surrogate light chains $(\mu / S L C)$. (b) Potential influence of lowered availability of arginine on $B$ cell development. The ratio of pre- to pro-B cells was decreased in arginase I transgenic mice compared to wild-type. It must be emphasized that expression of $\mu / S L C$ in large pre-B cells was not determined. However, the mRNA levels of stromal-cell derived factor-1 (SDF-1) and IL-7, and maturation beyond the pro-B to pre-B cell transition were not affected, or much less affected, by the reduced availability of arginine. Apoptotic events or a reduction in available survival factors produced by BM stromal cells may influence the transition from pro-B to large pre-B cells during reduced arginine availability.

The identity of the arginine-sensitive target cell(s) was also not elucidated. An arginine-dependent metabolic requirement (e.g., synthesis of argininerich proteins) unique to pro-B or pre-B cells (vis-à-vis other lymphohematopoietic cells) is difficult to imagine. It seems more probable that a decrease in arginine availability in the $\mathrm{BM}$ microenvironment may have modified gene expression in the pro-B/pre$\mathrm{B}$ cells, or a stromal cell component that supports B cell development (Figure 1). Amino acid regulation of gene expression can occur at the level of transcription, translation, or protein stability (10). In addition to a direct analysis of arginase I transgenics, experiments testing the role of arginine in proliferation and/or differentiation of normal murine $\mathrm{B}$ cell precursors or cell lines may prove fruitful. It should be noted that a simple cell culture model was employed to demonstrate a role for arginine in stabilizing the $\mathrm{CD} 3$ $\zeta$ subunit of the $T$ cell receptor (8).

A decrease in arginine availability may also modify gene expression in a stromal cell component. The authors used RT-PCR analysis of total BM to show there was no difference in expression of IL-7, the IL-7 receptor complex (IL-7 receptor $\alpha$ subunit and the common $\gamma$ subunit), stromal cell-derived factor-1 (SDF-1), or CXCR4 (the receptor for SDF-1) in arginase I transgenics versus normal littermates (Figure 1). However, a reduced capacity of SDF-1 or IL-7 (or some other cytokine) to be synthesized, secreted, and/or associated with the ECM could contribute to the developmental block. It is also conceivable that arginine deficiency in BM stromal cells suppressed the expression of a gene (or genes) that encodes a pre$B$ cell survival factor, or activated the expression of a gene (or genes) encoding a molecule that promotes apopto- sis in pre-B cells (Figure 1). One problem with a BM stromal cell as the arginine-sensitive target would be the requirement that a $B$ lineage cell-specific supportive function be compromised, i.e., stromal cell arginine deficiency would only alter expression of gene products that uniquely regulate $B$ cell development. BM stromal cell niches that support B cell development probably support myelopoiesis as well. Thus, although a detailed analysis of myelopoiesis in arginase I transgenics was not conducted $(6,9)$, an affect restricted to a BM stromal cell element seems unlikely.

Does the study by de Jonge and colleagues provide any food for thought regarding a comparable requirement for arginine in human $\mathrm{B}$ cell development? There are no published reports ascribing any unique role for arginine in human pro-B/pre-B cell survival or differentiation. Models are available for studying human B cell differentiation $(11,12)$ and a role for arginine could be tested. Increased plasma concentrations of arginase I, and a concomitant decrease in plasma arginine concentrations, have been described in liver transplant recipients and patients with some tumors (discussed in refs. 6 and 8 ). Whether or not these patients had alterations in B cell development is unknown. The possibility exists that reduced arginine levels could contribute to the phenotype of patients with antibody deficiency diseases. In newborn humans, the dietary supply of arginine derived from milk is insufficient to meet the minimum requirement necessary for incorporation of arginine into the total protein pool (7). Thus, endogenous arginine biosynthesis is important in the neonatal period. Patients with mutations in Btk (giving rise to classic $\mathrm{X}$-linked agammaglobulinemia, Ig- $\alpha, \lambda 5$, or BLNK all have a block at the pro- $\mathrm{B}$ to pre- $\mathrm{B}$ stage of $\mathrm{B}$ cell development (13). It is therefore conceivable that a deficiency in arginine could contribute to the severity of the block in pre-B cell development. This possibility could be tested by crossing arginase I transgenic mice with, for example, xid (X-linked immunodeficient) mice harboring a mutation in Btk. Given that mice with defects in the pre-BCR signaling pathway have milder forms of $B$ cell immunodeficiency compared to humans (13), a more severe disruption 
in B cell development may occur in arginase $I /$ xid double transgenics.

In conclusion, determination of the significance of a transient reduction in the availability of a single amino acid on mammalian lymphocyte development in health and disease will require additional work.

1. Hardy, R.R., and Hayakawa, K. 2001. B cell development pathways. Annu. Rev. Immunol. 19:595-621.

2. LeBien, T.W. 2000. Fates of human B-cell precursors. Blood. 96:9-23.

3. Meffre, E., Casellas, R., and Nussenzweig, M.C. 2000. Antibody regulation of B cell development. Nat. Immunol. 1:379-385.
4. Glimcher, L.H., and Singh, H. 1999. Transcription factors in lymphocyte development - $\mathrm{T}$ and B cells get together. Cell. 96:13-23.

5. Kincade, P.W., et al. 1998. Life/death decisions in B lymphocyte precursors: a role for cytokines, cell interaction molecules, and hormones. In Molecular biology of B-cell and T-cell development. J. Monroe and E. Rothenberg, editors. Humana Press. Totowa, New Jersey, USA. 177-196.

6. de Jonge, W.J., et al. 2002. Arginine deficiency affects early B cell maturation and lymphoid organ development in transgenic mice. J. Clin. Invest. 110:1539-1548. doi:10.1172/JCI200216143.

7. Wu, G., and Morris, S.M., Jr. 1998. Arginine metabolism: nitric oxide and beyond. Biochem. J. 336:1-17.

8. Rodriguez, P.C., et al. 2002. Regulation of T cell receptor $\mathrm{CD} 3 \zeta$ chain expression by L-arginine. J. Biol. Chem. 227:21123-21129.

9. de Jonge, W.J., et al. 2002. Overexpression of arginase I in enterocytes of transgenic mice elic its a selective arginine deficiency and affects skin, muscle, and lymphoid development. Am. J. Clin. Nutr. 76:128-140.

10. Fafournoux, P., Bruhat, A., and Jousse, C. 2000 Amino acid regulation of gene expression. Biochem. J. 351:1-12.

11. Villablanca,J.G., et al. 1990. Differentiation of normal human pre-B cells in vitro. J. Exp. Med. 172:325-334.

12. Bertrand, F.E., Vogtenhuber, C., Shah, N., and LeBien, T.W. 2001. Pro-B cell to pre-B cell development in B-lineage acute lymphoblastic leukemia expressing the MLL/AF4 fusion protein. Blood. 98:3398-3405.

13. Conley, M.E., Rohrer, J., Rapalus, L., Boylin, E.C., and Minegishi, Y. 2000. Defects in early B-cell development: comparing the consequences of abnormalities in pre-BCR signaling in the human and the mouse. Immunol. Rev. 178:75-90. 